\title{
ON A GENERALIZATION OF HILBERT'S DOUBLE SERIES THEOREM
}

\author{
BICHENG YANG
}

Abstract. By introducing three parameters $A, B$ and $\lambda$, we give a generalization of Hilbert's inequality with a best constant factor and some particular results.

Mathematics subject classification (2000): 26D15.

Key words and phrases: Hilbert's inequality, weight coefficient, $\beta$ function, Cauchy's inequality.

\section{REFERENCES}

[1] Hardy G. H., Littlewood J. E. and Polya G., Inequalities. Cambridge, Cambridge Univ. Press (1952).

[2] Hu KE, On Hilbert inequality and its application, Advances in Mathematics 22 (1993), 160-163.

[3] YANG BICHENG, A refinement of Hilbert's inequality, Huanghuai J. 13 (2) (1997), 47-51.

[4] GaO MingZHE, On Hilbert's inequality and its applications, J. Math. Anal. Appl. 212 (1997), $316-323$.

[5] YANG BICHENG AND DEBNATH L., On a new generalization of Hardy-Hilbert's inequality and its applications, J. Math. Anal. Appl. 233 (1999), 484-497.

[6] KANG JiChANG AND DEBNATH L., On a new generalization of Hardy-Hilbert's inequality and their applications, J. Math. Anal. Appl. 245 (2000), 248-265.

[7] Gao Mingzhe, Tan Li And DeBnath L., Some improvements on Hilbert's integral inequality, J. Math. Anal. Appl. 229 (1999), 682-689.

[8] YAng Bicheng, On Hilbert's integral inequality, J. Math. Anal. Appl. 220 (1998), 778-785.

[9] YANG Bicheng, A note on Hilbert's integral inequalities, Chin Quart J. Math. 13 (4) (1998), 83-86.

[10] Hsu L. C. AND Wang Y. J., A refinement of Hilbert's double theorem, J. Math. Res. \& Exp. 11 (1) (1991), 143-144.

[11] Gao Mingzhe, A note on Hilbert double theorem, Hunan Anna. Math. 12 (1-2) (1992), $142-147$.

[12] YANG BICHENG, On new generalizations of Hilbert's integral, J. Math. Anal. Appl. 248 (2000), $29-40$. 